\title{
ROBUST CONTROLLED POSITIVE DELAYED SYSTEMS WITH INTERVAL PARAMETER UNCERTAINTIES: A DELAY UNIFORM DECOMPOSITION APPROACH
}

\author{
WAFA Elloumi $^{a, *}$, Driss MEHDI $^{b}$, MOHAMEd CHAABANE $^{a}$ \\ ${ }^{a}$ Laboratory of Sciences and Techniques of Automatic Control and Computer Engineering (Lab-STA) \\ University of Sfax, PB 1173, 3038 Sfax, Tunisia \\ e-mail: elloumi.wafa@gmail.com chaabane_uca@yahoo.fr \\ ${ }^{b}$ Laboratory of Computer Science and Automatic Control for Systems (LIAS/ENSIP) \\ University of Poitiers, 2, rue Pierre Brousse, 86073 Poitiers Cedex 9, France \\ e-mail: driss.mehdi@univ-poitiers.fr
}

\begin{abstract}
This paper is concerned with robust stabilization of continuous linear positive time-delay systems with parametric uncertainties. The delay considered in this work is a bounded time-varying function. Previously, we have demonstrated that the equidistant delay-decomposition technique is less conservative when it is applied to linear positive time-delay systems. Thus, we use simply a delay bi-decomposition in an appropriate Lyapunov-Krasovskii functional. By using classical and partitioned control gains, the state-feedback controllers developed in our work are formulated in terms of linear matrix inequalities. The efficiency of the proposed robust control laws is illustrated with via an example.
\end{abstract}

Keywords: delay systems, robust stabilization, positive systems, parametric constraints, delay decomposition, LMIs.

\section{Introduction}

Positive systems are largely encountered in many real process (biology, statistics, thermodynamics, ecology, networking, etc.). Accordingly, many researchers are continuously interested in these systems (Luenberger, 1976; Shorten et al., 2006; Zhang and Yang, 2013; Kaczorek, 2014; 2016; Shuqian et al., 2014; Junfeng et al., 2017). Starting from a nonnegative initial state, the key mathematical property of positive systems is the state evolution in the positive orthant for all nonnegative inputs. Designing control laws in such a way that the closed-loop system is positive and robustly stable when there are parametric uncertainties is a topic of continuing interest in the literature (Mesquine et al., 2015; Shuqian et al., 2014; Zaidi et al., 2014; Hmamed et al., 2012; Bolajraf, 2012). When the system dynamics are also influenced by a time delay, the problem of robust stabilization becomes more complicated. To tackle the issue, Zaidi et al. (2014), Hmamed et al. (2012) and Bolajraf (2012) discussedrelated to state-feedback

* Corresponding author asymptotic stabilization under the positivity constraint for positive delayed systems by using linear matrix inequalities (LMIs) and linear programming (LP).

It should be mentioned that in some cases of positive systems' the stability conditions may depend on the time delay, in particular, in the cases of stochastic stability for positive discrete-time Markov jump linear systems (Zhu et al., 2016; 2017) and the exponential stability of positive systems with constant and time-varying delay (Zhu et al., 2013). In this work, we are interested in the exponential stability and stabilization known for its effect on the faster convergence of states. In the other hand, we are motivated by the result presented by Elloumi et al. (2015) demonstrating that increasing the delay decomposition provides less conservative results. Unfortunately, this leads to solving a large-scale system of LMIs when faced with a large number of delay decomposition. Thus, we use simply a bi-decomposition technique to establish the state-feedback controller.

The main contribution of this paper concerns the robust $\alpha$-exponential stabilization for continuous positive systems with time-varying delay when the control design 
might include parametric uncertainties. The proposed approach is based on the exponential stability conditions proposed by Elloumi et al. (2015), especially those founded by an equidistant bi-decomposition of the delay. By using Lyapunov-Krasovskii functions (LKFs), the elaborated conditions can easily be solved by using LMIs.

The remainder of the paper is structured as follows: Section 2 is devoted to the problem formulation while some exploratory results about the exponential stability of positive delayed systems are presented in Section 3. The state-feedback stabilization problem without and with parametric uncertainties is investigated in Section 4. Then, in Section 5, the achieved result related to the robust exponential stabilization is relaxed. Section 6 covers an illustrative example to highlight the efficiency of the proposed control laws. Finally, we end with concluding remarks in Section 6.

In the sequal, we use the following notation: $\mathbb{R}_{+}^{n}$ stands for the non-negative orthant of the $n$-dimensional real space $\mathbb{R}^{n}$. Let $M$ be a matrix (or a vector). If all the components of $M$ are nonnegative, then $M$ is said to be nonnegative (written $M \succeq 0$ ). Meanwhile, if all its components are positive, it is said to be positive ( $M \succ 0$ ). A matrix $M \in \mathbb{R}^{n \times n}$ is called a Metzler one if all the elements of its off-diagonal are nonnegative. That is, if $M=\left\{m_{i j}\right\}_{i, j=1}^{n}$, if $m_{i j} \succ 0$ when $i \neq j, M$ is Metzler. A matrix $M \in \mathbb{R}^{n \times n}$ is called an $M$-matrix if and only if there exists a positive vector $\lambda$ such that $M \lambda \succ 0$. The condition that a matrix $P \in \mathbb{R}^{n \times m}$ positive definite is written as $P>0$. Let $\phi(t)$ be a function defined on the interval $[0, \tau]$. The norm $\|\phi\|_{f}$ is given by

$$
\|\phi\|_{f}=\max _{\tau \leq \theta \leq 0}\{\|x(t+\theta)\|,\|\dot{x}(t+\theta)\|\} .
$$

\section{Problem formulation}

Consider the continuous-time delayed linear system governed by

$$
\left\{\begin{array}{l}
\dot{x}(t)=A x(t)+A_{1} x(t-h(t))+B u(t) \\
x(t)=\phi(t) \succeq 0, \quad t \in\left[-h_{M}, 0\right]
\end{array}\right.
$$

where $x \in \mathbb{R}^{n}$ represents the state vector and $u \in$ $\mathbb{R}^{m}$ is the control vector. Moreover, we consider the time-varying delay $h(t) \in \mathbb{R}$ as a continuous and bounded function defined by

$$
\begin{aligned}
h_{m}=0 \preceq h(t) & \preceq h_{M}, \\
\dot{h}(t) & \preceq d .
\end{aligned}
$$

The matrices $A \in \mathbb{R}^{n \times n}, A_{1} \in \mathbb{R}^{n \times n}$ and $B \in \mathbb{R}^{n \times m}$ of the system (1) are assumed to be unknown and bounded by know constant matrices $\underline{A}, \bar{A}, \underline{A} 1, \bar{A}_{1}, \underline{B}$ and $\bar{B}$, as follows:

$$
\begin{gathered}
\underline{A} \leq A \leq \bar{A}, \\
0 \preceq \underline{A}_{1} \leq A_{1} \leq \bar{A}_{1}, \\
\underline{B} \leq B \leq \bar{B} .
\end{gathered}
$$

In the literature, many researchers (Rami, 2011; Hmamed et al., 2012; Chen et al., 2017) were interested in the class of uncertainties (3) which are called interval uncertainties since the parameters (i.e., $A, A_{1}$ and $B$ ) vary over intervals.

Matrix $A_{1}$ in (1) is a nonnegative matrix while there is no requirement that matrix $A$ be Metzler. To ensure the positivity and the exponential stability of the closed-loop system, one only has to use the following memoryless state feedback law:

$$
u(t)=K x(t) .
$$

By using the state-feedback control (4), the obtained closed-loop system is

$$
\left\{\begin{array}{l}
\dot{x}(t)=(A+B K) x(t)+A_{1} x(t-h(t)), \\
x(t)=\phi(t) \succeq 0, \quad t \in\left[-h_{M}, 0\right] .
\end{array}\right.
$$

In this paper, we aim at establishing a state feedback controller in the form of (4) for the continuous linear time-delay system (5) without and with parametric interval uncertainties in such a way that the resulting closed-loop system is positive and $\alpha$-exponentially stable.

Note that in the problem addressed in this paper, $A_{1}$ is a nonnegative matrix with no restrictions on $A$. But, if there are no restrictions on both of $A$ and $A_{1}$ and the delay $h(t)$ is known at all times, the above objectives are reached by using the memory control law $u(t)=K x(t)+$ $F x(t-h(t))$.

\section{Preliminaries}

First of all, we define the linear autonomous delayed system

$$
\left\{\begin{array}{l}
\dot{x}(t)=A x(t)+A_{1} x(t-h(t)), \\
x(t)=\phi(t) \succeq 0, \quad t \in\left[-h_{M}, 0\right],
\end{array}\right.
$$

where $A$ is a Metzler matrix and $A_{1} \succeq 0$.

Definition 1. (Hale and Lunel, 1993) Given $\alpha>0$ the zero solution of system (6) is exponentially stable with a decay rate $\alpha$ if there exists a positive number $S \succ 0$ such that every solution $x(t, \phi)$ satisfies

$$
\|x(t, \phi)\| \preceq S e^{-\alpha t}\|\phi\|, \quad t \in \mathbb{R}_{+} .
$$

The previous definition is concerned with the exponential stability of the system (6). The following definition is about its positivity. 
Definition 2. (Farina and Rinaldi, 2000) Given any positive initial condition $x(t)=\phi(t) \in \mathbb{R}_{+}^{n}, t \in$ $\left[-h_{M}, 0\right]$, the delayed system (6) is said to be positive if the corresponding trajectory is never negative, i.e., $x(t) \in$ $\mathbb{R}_{+}^{n}$ for all $t \geq 0$

Based on Definition 2, we look for conditions on which the delayed system (22) is positive.

Lemma 1. (Farina and Rinaldi, 2000) System (6) is positive (i.e., $x(t) \in \mathbb{R}_{+}^{n}$ ) if and only if $A$ is a Metzler matrix and $A_{1}$ is a nonnegative matrix.

Lemma 2. (Luenberger, 1976) Matrix $M$ is Metzler if and only if there exists a positive scalar $\gamma$ such that

$$
M+\gamma I \succ 0 .
$$

Lemma 3. (Araki, 1975) Let $M$ be a Metzler matrix. Then $-M$ is an M-matrix if and only if there is a positive definite matrix $W$ such that matrix $M^{\top} W+W M$ is negative definite.

In the following, we recall sufficient conditions for the $\alpha$-exponential stability of the linear positive delayed system (6) by using a technique of delay uniform bi-decomposition.

Lemma 4. (Elloumi et al., 2015) For some given scalars $h_{M}, \alpha>0$ and $h_{a}\left(h_{a}=h_{M} / 2\right)$, if there exist positive definite matrices $Q_{3}, P, Z_{i},(i=1,2,3)$ and $Q_{1}=$ $Q_{1}^{\top}, Q_{12}, Q_{2}=Q_{2}^{\top}$ with appropriate dimensions, system (6) is simultaneously positive and exponentially stable, where $\alpha$ is defined as its decay rate for $h(t)$ satisfying (2), such that

$$
\begin{gathered}
Q=\left[\begin{array}{cc}
Q_{1} & Q_{12} \\
Q_{12}^{\top} & Q_{2}
\end{array}\right] \geq 0, \\
\Psi_{i}<0, \quad i=1,2,
\end{gathered}
$$

where

$$
\begin{aligned}
\Psi_{1}= & {\left[\begin{array}{ccccc}
\Psi_{11} & \Psi_{12}^{1} & \Psi_{13}^{1} & 0 & A^{\top} E_{1} \\
* & \Psi_{22}^{1} & \Psi_{23}^{1} & 0 & A_{1}^{\top} E_{1} \\
* & * & \Psi_{33}^{1} & \Psi_{34}^{1} & 0 \\
* & * & * & \Psi_{44}^{1} & 0 \\
* & * & * & * & -E_{1}
\end{array}\right], } \\
\Psi_{2}= & {\left[\begin{array}{ccccc}
\Psi_{11} & P A_{1} & \Psi_{13}^{2} & 0 & A^{\top} E_{2} \\
* & \Psi_{22}^{2} & \Psi_{23}^{2} & \Psi_{24}^{2} & A_{1}^{\top} E_{2} \\
* & * & \Psi_{33}^{2} & \Psi_{34}^{2} & 0 \\
* & * & * & \Psi_{44}^{2} & 0 \\
* & * & * & * & -E_{2}
\end{array}\right], }
\end{aligned}
$$

$$
\begin{aligned}
& \Psi_{11}=2 \alpha P+Q_{1}+Q_{3}+P A+A^{\top} P \\
& -\frac{e^{-2 \alpha h_{a}}}{h_{a}}\left(Z_{1}+(1-d) Z_{3}\right), \\
& \Psi_{12}^{1}=P A_{1}+\frac{e^{-2 \alpha h_{a}}}{h_{a}}\left(Z_{1}+(1-d) Z_{3}\right), \\
& \Psi_{13}^{1}=Q_{12}, \\
& \Psi_{22}^{1}=-e^{-2 \alpha h_{a}}(1-d) Q_{3}-\frac{e^{-2 \alpha h_{a}}}{h_{a}} Z_{1} \\
& -\frac{e^{-2 \alpha h_{a}}}{h_{a}}\left(Z_{1}+(1-d) Z_{3}\right), \\
& \Psi_{23}^{1}=\frac{e^{-2 \alpha h_{a}}}{h_{a}} Z_{1}, \\
& \Psi_{33}^{1}=-e^{-2 \alpha h_{a}} Q_{1}+Q_{2}-\frac{e^{-2 \alpha h_{a}}}{h_{a}} Z_{1} \\
& -\frac{e^{-2 \alpha h_{M}}}{h_{a}} Z_{2}, \\
& \Psi_{34}^{1}=\frac{e^{-2 \alpha h_{M}}}{h_{a}} Z_{2}-e^{-2 \alpha h_{a}} Q_{12}, \\
& \Psi_{44}^{1}=-e^{-2 \alpha h_{a}} Q_{2}-\frac{e^{-2 \alpha h_{M}}}{h_{a}} Z_{2}, \\
& \Psi_{13}^{2}=Q_{12}+\frac{e^{-2 \alpha h_{a}}}{h_{a}}\left(Z_{1}+(1-d) Z_{3}\right) \\
& \Psi_{22}^{2}=-e^{-2 \alpha h_{M}}(1-d) Q_{3}-\frac{e^{-2 \alpha h_{M}}}{h_{a}} Z_{2}, \\
& -\frac{e^{-2 \alpha h_{M}}}{h_{a}}\left(Z_{2}+(1-d) Z_{3}\right), \\
& \Psi_{23}^{2}=\frac{e^{-2 \alpha h_{M}}}{h_{a}}\left(Z_{2}+(1-d) Z_{3}\right), \\
& \Psi_{24}^{2}=\frac{e^{-2 \alpha h_{M}}}{h_{a}} Z_{2} \\
& \Psi_{33}^{2}=-e^{-2 \alpha h_{a}} Q_{1}+Q_{2}-\frac{e^{-2 \alpha h_{a}}}{h_{a}}\left(Z_{1}+(1-d) Z_{3}\right), \\
& -\frac{e^{-2 \alpha h_{M}}}{h_{a}}\left(Z_{2}+(1-d) Z_{3}\right), \\
& \Psi_{34}^{2}=-e^{-2 \alpha h_{a}} Q_{12}, \\
& \Psi_{44}^{2}=-e^{-2 \alpha h_{a}} Q_{2}-\frac{e^{-2 \alpha h_{M}}}{h_{a}} Z_{2}, \\
& E_{1}=h_{a} Z_{1}+\left(h_{M}-h_{a}\right) Z_{2}+h_{a} Z_{3}, \\
& E_{2}=h_{a} Z_{1}+\left(h_{M}-h_{a}\right) Z_{2}+h_{M} Z_{3} .
\end{aligned}
$$

In addition, the solution of the system should satisfy

$$
\|x\| \preceq \sqrt{\frac{b}{a}} \times e^{-\alpha t}\|\phi(t)\|, \quad t \geq 0,
$$

where

$$
\begin{aligned}
a= & \lambda_{\min }(P) \\
b= & {\left[\lambda_{\max }[P]+\lambda_{\max }\left[Q_{3}\right]\right.} \\
& +\lambda_{\max }\left[Q_{1}\right]+\lambda_{\max }\left[Q_{2}\right]+2 \lambda_{\max }\left[Q_{12}\right]
\end{aligned}
$$




$$
\begin{aligned}
& +h_{M}\left(\lambda_{\max }\left[Z_{1}\right]+\lambda_{\max }\left[Z_{2}\right]\right. \\
& \left.\left.+\lambda_{\max }\left[Z_{3}\right]\right)\right]\|\phi\|_{f}^{2} \frac{1-e^{-2 \alpha h_{M}}}{2 \alpha}
\end{aligned}
$$

Remark 1. Theorem 4 is based on two conditions formulated in terms of LMIs. This is due to the fact that we use the bi-decomposition of the interval $\left[0, h_{M}\right]$. Thus, as demonstrated by Elloumi et al. (2015), conservatism can be reduced by increasing the decomposition of $\left[0, h_{M}\right]$ although the number of LMIs is then greater.

\section{State-feedback exponential stabilization}

Based on Theorem 4, this section provides sufficient conditions for solving the problem of exponential stabilization by using a state-feedback law control $u(t)=$ $K x(t)$, leading to the closed-loop system

$$
\left\{\begin{array}{l}
\dot{x}(t)=(A+B K) x(t)+A_{1} x(t-h(t)), \\
x(t)=\phi(t) \succeq 0, \quad t \in\left[-h_{M}, 0\right]
\end{array}\right.
$$

where the matrix $K \in \mathbb{R}^{m \times n}$ is selected through the following problem: Find sufficient conditions on matrices $A, A_{1} \in \mathbb{R}^{n \times n}, B \in \mathbb{R}^{n \times m}$, such that there exists a matrix $K \in \mathbb{R}^{m \times n}$ that guarantees

- positivity in closed-loop $\left(A_{c}=A+B K\right.$ is a Metzler matrix), and

- closed-loop $\alpha$-exponential stability.

Theorem 1. Having a time-varying delay in the form (2), the linear continuous system (12) is $\alpha$-exponentially stable and positive for given scalars $\alpha \succ 0, h_{M}, h_{a}$ and $\gamma \succ 0$, if there exist a diagonal positive matrix $X$, a matrix $Y$ and positive definite matrices $\bar{Q}_{i}$ and $\bar{Z}_{i},(i=1,2,3)$ with appropriate dimensions, such that

$$
\begin{gathered}
\bar{\Psi}_{i}<0, \quad i=1,2, \\
A X+B Y+\gamma X \succ 0,
\end{gathered}
$$

where

$$
\begin{aligned}
\bar{\Psi}_{1} & =\left[\begin{array}{ccccc}
\bar{\Psi}_{11} & \bar{\Psi}_{12}^{1} & \bar{\Psi}_{13}^{1} & 0 & X A^{\top}+Y^{\top} B^{\top} \\
* & \bar{\Psi}_{22}^{1} & \bar{\Psi}_{23}^{1} & 0 & X A_{1}^{\top} \\
* & * & \bar{\Psi}_{33}^{1} & \bar{\Psi}_{34}^{1} & 0 \\
* & * & * & \bar{\Psi}_{44}^{1} & 0 \\
* & * & * & * & -\left(2 X-\bar{E}_{1}\right)
\end{array}\right] \\
& <0
\end{aligned}
$$$$
\bar{\Psi}_{2}=\left[\begin{array}{ccccc}
\bar{\Psi}_{11} & A_{1} X & \bar{\Psi}_{13}^{2} & 0 & X A^{\top}+Y^{\top} B^{\top} \\
* & \bar{\Psi}_{22}^{2} & \bar{\Psi}_{23}^{2} & \bar{\Psi}_{24}^{2} & X A_{1}^{\top} \\
* & * & \bar{\Psi}_{33}^{2} & \bar{\Psi}_{34}^{2} & 0 \\
* & * & * & \bar{\Psi}_{44}^{2} & 0 \\
* & * & * & * & -\left(2 X-\bar{E}_{2}\right)
\end{array}\right]
$$$$
<0 \text {, }
$$

$$
\begin{aligned}
& \bar{\Psi}_{11}=2 \alpha X+\bar{Q}_{1}+\bar{Q}_{3}+A X+X A^{\top}+B Y \\
& +Y^{\top} B^{\top}-\frac{e^{-2 \alpha h_{a}}}{h_{a}}\left(\bar{Z}_{1}+(1-d) \bar{Z}_{3}\right), \\
& \bar{\Psi}_{12}^{1}=A_{1} X+\frac{e^{-2 \alpha h_{a}}}{h_{a}}\left(\bar{Z}_{1}+(1-d) \bar{Z}_{3}\right), \\
& \bar{\Psi}_{13}^{1}=\bar{Q}_{12}, \\
& \bar{\Psi}_{22}^{1}=-e^{-2 \alpha h_{a}}(1-d) \bar{Q}_{3}-\frac{e^{-2 \alpha h_{a}}}{h_{a}} \bar{Z}_{1} \\
& -\frac{e^{-2 \alpha h_{a}}}{h_{a}}\left(\bar{Z}_{1}+(1-d) \bar{Z}_{3}\right), \\
& \bar{\Psi}_{23}^{1}=\frac{e^{-2 \alpha h_{a}}}{h_{a}} \bar{Z}_{1}, \\
& \bar{\Psi}_{33}^{1}=-e^{-2 \alpha h_{a}} \bar{Q}_{1}+\bar{Q}_{2} \\
& -\frac{e^{-2 \alpha h_{a}}}{h_{a}} \bar{Z}_{1}-\frac{e^{-2 \alpha h_{M}}}{h_{a}} \bar{Z}_{2}, \\
& \bar{\Psi}_{34}^{1}=\frac{e^{-2 \alpha h_{M}}}{h_{a}} \bar{Z}_{2}-e^{-2 \alpha h_{a}} Q_{12}, \\
& \bar{\Psi}_{44}^{1}=-e^{-2 \alpha h_{a}} \bar{Q}_{2}-\frac{e^{-2 \alpha h_{M}}}{h_{a}} \bar{Z}_{2}, \\
& \bar{\Psi}_{13}^{2}=\bar{Q}_{12}+\frac{e^{-2 \alpha h_{a}}}{h_{a}}\left(\bar{Z}_{1}+(1-d) \bar{Z}_{3}\right), \\
& \bar{\Psi}_{22}^{2}=-e^{-2 \alpha h_{M}}(1-d) \bar{Q}_{3}-\frac{e^{-2 \alpha h_{M}}}{h_{a}} \bar{Z}_{2} \\
& -\frac{e^{-2 \alpha h_{M}}}{h_{a}}\left(\bar{Z}_{2}+(1-d) \bar{Z}_{3}\right), \\
& \bar{\Psi}_{23}^{2}=\frac{e^{-2 \alpha h_{M}}}{h_{a}}\left(\bar{Z}_{2}+(1-d) \bar{Z}_{3}\right), \\
& \bar{\Psi}_{24}^{2}=\frac{e^{-2 \alpha h_{M}}}{h_{a}} \bar{Z}_{2}, \\
& \bar{\Psi}_{33}^{2}=-e^{-2 \alpha h_{a}} \bar{Q}_{1}+\bar{Q}_{2} \\
& -\frac{e^{-2 \alpha h_{a}}}{h_{a}}\left(\bar{Z}_{1}+(1-d) \bar{Z}_{3}\right) \\
& -\frac{e^{-2 \alpha h_{M}}}{h_{a}}\left(\bar{Z}_{2}+(1-d) \bar{Z}_{3}\right), \\
& \bar{\Psi}_{34}^{2}=-e^{-2 \alpha h_{a}} \bar{Q}_{12}, \\
& \bar{\Psi}_{44}^{2}=-e^{-2 \alpha h_{a}} \bar{Q}_{2}-\frac{e^{-2 \alpha h_{M}}}{h_{a}} \bar{Z}_{2}, \\
& \bar{E}_{1}=h_{a} \bar{Z}_{1}+\left(h_{M}-h_{a}\right) \bar{Z}_{2}+h_{a} \bar{Z}_{3}, \\
& \bar{E}_{2}=h_{a} \bar{Z}_{1}+\left(h_{M}-h_{a}\right) \bar{Z}_{2}+h_{M} \bar{Z}_{3} \text {. }
\end{aligned}
$$

Proof. The $\alpha$-exponential stabilitization condition (13) is derived by applying the following steps. First, pre- and post-multiply the matrix $\hat{X}=\operatorname{diag}\left\{X, X, X, X, E_{1}^{-1}\right\}$ by the first LMI of (8). Similarly, pre- and post-multiply the second LMI of 8 by the matrix $\hat{X}=$ $\operatorname{diag}\left\{X, X, X, X, E_{2}^{-1}\right\}$. Then, each of matrices $K, A$, $P$ and $E_{i}(i=1,2)$ is replaced by $Y X^{-1}, A+B K, X^{-1}$ 
and $X^{-1} \overline{E_{i}} X^{-1}$, respectively. Consequently, we get

$$
\begin{aligned}
\bar{\Psi}_{1}= & {\left[\begin{array}{ccccc}
\bar{\Psi}_{11} & \bar{\Psi}_{12}^{1} & 0 & 0 & X A_{c}^{\top} \\
* & \bar{\Psi}_{22}^{1} & \bar{\Psi}_{23}^{1} & 0 & X A_{1}^{\top} \\
* & * & \bar{\Psi}_{33}^{1} & \bar{\Psi}_{34}^{1} & 0 \\
* & * & * & \bar{\Psi}_{44}^{1} & 0 \\
* & * & * & * & -X \bar{E}_{1}^{-1} X
\end{array}\right]<0, } \\
\bar{\Psi}_{2}= & {\left[\begin{array}{ccccc}
\bar{\Psi}_{11} & A_{1} X & \bar{\Psi}_{13}^{2} & 0 & X A_{c}^{\top} \\
* & \bar{\Psi}_{22}^{2} & \bar{\Psi}_{23}^{2} & \bar{\Psi}_{24}^{2} & X A_{1}^{\top} \\
* & * & \bar{\Psi}_{33}^{2} & 0 & 0 \\
* & * & * & \bar{\Psi}_{44}^{2} & 0 \\
* & * & * & * & -X \bar{E}_{2}^{-1} X
\end{array}\right]<0 . }
\end{aligned}
$$

Since $\left(X-\bar{E}_{i}\right) \bar{E}_{i}^{-1}\left(X-\bar{E}_{i}\right)>0, i=1,2$, we deduce that $-X \bar{E}_{i}^{-1} X<-2 X+\bar{E}_{i}$. Finally, all the terms $X \Psi_{i j} X$, for $i, j=1, \ldots, 5$ are replaced by $\bar{\Psi}_{i j}$. The same operation is applied to the matrices $Q_{i}$ and $Z_{i}$ for $i=1, \ldots, 3$. Thus, the LMI (13) in Theorem 1 is satisfied. Consequently, the $\alpha$-exponential stabilization condition of the closed-loop delay system (12) is ensured with the state-feedback control $u(t)=K x(t)$.

The proof of the positivity condition (14) is mainly based on the idea of Theorem 3 . Assume that condition (14) is satisfied. Since matrix $X$ is diagonal and positive, the inverse matrix $X^{-1}$ is also diagonal and positive. Then post-multiplication by $X^{-1}$ is applied to the LMI (14). Thus, for $\gamma \succ 0$, we get $A+B Y X^{-1}+\gamma I \succ 0$, which leads to $A+B K+\gamma I \succ 0$. Consequently, by using Lemma 2 $A+B K$ is a Metzler matrix. Indeed, $A_{1}$ and $B$ are assumed to be non negative matrices, which means that the closed-loop system is also positive.

Remark 2. The LMIs proposed in Theorem 1 are efficient to ensure the positivity and the exponential stability in the closed-loop of the class of linear continuous systems defined by (12). However, this may not be guaranteed when there is a parametric variation.

4.1. Robust exponential stabilization. In order to extend the result presented in the previous section, this section is concerned with the robust stabilization of delayed system mathematically described by (1) when it subject to interval uncertainties (3). Therefore, we retain the same control law form defined by

$$
u(t)=K x(t) .
$$

The resulting continuous delayed system is

$$
\left\{\begin{array}{l}
\dot{x}(t)=(A+B K) x(t)+A_{1} x(t-h(t)), \\
\underline{A} \leq A \leq \bar{A}, \\
0 \preceq \underline{A}_{1} \leq A_{1} \leq \bar{A}_{1}, \\
\underline{B} \leq B \leq \bar{B} .
\end{array}\right.
$$

Corollary 1. Having a control law in the form of (15), the closed-loop system (16) is $\alpha$-exponentially stable and positive with parametric uncertainties defined by (3) and a delay function $h(t)$ satisfying (2), if, for given scalars $h_{M}, \alpha \succ 0, h_{a}\left(h_{a}=h_{M} / 2\right)$ and $\gamma \succ 0$, there exist positive definite matrices $\bar{Q}_{i}$ and $\bar{Z}_{i}(i=1,2,3)$, a positive diagonal matrix $X$ with appropriate dimensions and a matrix $Y \in \mathbb{R}^{m \times n}$ such that

$$
\begin{gathered}
\bar{\Psi}_{i *}<0, \quad i=1,2, \\
\underline{A} X+\underline{B} Y+\gamma X \succ 0,
\end{gathered}
$$

where

$$
\begin{aligned}
& \bar{\Psi}_{1 *}=\left[\begin{array}{ccccc}
\bar{\Psi}_{11 *} & \bar{\Psi}_{12}^{1} & \bar{\Psi}_{13}^{1} & 0 & \bar{\Psi}_{15}^{1 *} \\
* & \bar{\Psi}_{22}^{1} & \bar{\Psi}_{23}^{1} & 0 & X \bar{A}_{1}^{\top} \\
* & * & \bar{\Psi}_{33}^{1} & \bar{\Psi}_{34}^{1} & 0 \\
* & * & * & \bar{\Psi}_{44}^{1} & 0 \\
* & * & * & * & -\left(2 X-\bar{E}_{1}\right)
\end{array}\right], \\
& \bar{\Psi}_{2 *}=\left[\begin{array}{ccccc}
\bar{\Psi}_{11 *} & \bar{A}_{1} & \bar{\Psi}_{13}^{2} & 0 & \bar{\Psi}_{15 *}^{2} \\
* & \bar{\Psi}_{22}^{2} & \bar{\Psi}_{23}^{2} & \bar{\Psi}_{24}^{2} & X \bar{A}_{1}^{\top} \\
* & * & \bar{\Psi}_{33}^{2} & \bar{\Psi}_{34}^{2} & 0 \\
* & * & * & \bar{\Psi}_{44}^{2} & 0 \\
* & * & * & * & -\left(2 X-\bar{E}_{2}\right)
\end{array}\right], \\
& \bar{\Psi}_{11 *}=2 \alpha X+\bar{Q}_{1}+\bar{Q}_{3}+\bar{A} X+X \bar{A}^{\top}+\bar{B} Y \\
& +Y^{\top} \bar{B}^{\top}-\frac{e^{-2 \alpha h_{a}}}{h_{a}}\left(\bar{Z}_{1}+(1-d) \bar{Z}_{3}\right), \\
& \bar{\Psi}_{15 *}^{1}=\bar{\Psi}_{15 *}^{2}=X \bar{A}^{\top}+Y^{\top} \bar{B}^{\top} \text {. }
\end{aligned}
$$

The controller gain is given by

$$
K=Y X^{-1} \text {. }
$$

Proof. In the first step, we use the fact that $\underline{A} \leq A \leq \bar{A}$, $\underline{A}_{1} \leq A_{1} \leq \bar{A}_{1}$ and $\underline{B} \leq B \leq \bar{B}$ imply $\bar{\Psi}_{i} \leq \bar{\Psi}_{i *}$. Therefore, if $\bar{\Psi}_{* i}<0$, then $\bar{\Psi}_{i}<0$ for $i=1,2$. Consequently, we have the $\alpha$-exponential stability of the resulting closed-loop system (16) by using the control law $u(t)=K x(t)$ under the interval parametric uncertainties.

In the second step, we use the idea of Theorem 3 in order to obtain the positivity condition (18). Let the LMI (18) hold. $X^{-1}$ is diagonal positive since so is $X$. Post-multiplication by $X^{-1}$ is applied to the LMI (18). Accordingly, we obtain

$$
\underline{A}+\underline{B} Y X^{-1}+\gamma I \succ 0 \text {. }
$$

Introducing the expression for the controller gain $K$ in (19), we obtain

$$
A+B K+\gamma I \geq \underline{A}+\underline{B} K+\gamma I \succ 0,
$$


for $\gamma \succ 0$. Moreover, based on Lemma 2 $\underline{A}+\underline{B} K$ and $A+B K$ are Metzler matrices again taking into account that $A_{1}$ and $B$ are assumed to be non-negative matrices. Consequently, the closed-loop system (16) with the interval parametric uncertainties, which is $\alpha$-exponentially stable is positive.

\section{Relaxed robust stabilization}

In the case of constrained control or parametric uncertainties, many researchers use the technique of the controller gain partitioning (Rami et al., 2007; Bolajraf, 2012; Zaidi, 2015). Thus, we assume that for any matrix $K$, it is obvious that there exist nonnegative matrices $K^{+}$ and $K^{-}$such that $K=K^{+}-K^{-}$.

Consequently, the control law (4) can be rewritten as

$$
u(t)=\left(K^{+}-K^{-}\right) x(t) .
$$

Using (21), the closed-loop system becomes

$$
\begin{aligned}
\dot{x}(t)= & \left(A+B K^{+}-B K^{-}\right) x(t) \\
& +A_{1} x(t-h(t)) .
\end{aligned}
$$

Theorem 2. For given scalars $h_{M}, \alpha \succ 0, h_{a}\left(h_{a}=\right.$ $\left.h_{M} / 2\right)$ and $\gamma \succ 0$, the closed-loop system (22) is $\alpha$ exponentially stable and positive with parametric uncertainties defined by (3) and a delay function $h(t)$ satisfying (2), if there exist positive definite matrices $\bar{Q}_{i}$ and $\bar{Z}_{i}$ $(i=1,2,3)$, a positive diagonal matrix $X$ with appropriate dimensions, $Y^{+}$et $Y^{-} \in \mathbb{R}_{+}^{m \times n}$ such that

$$
\begin{gathered}
\overline{\bar{\Psi}}_{i}<0, \quad i=1,2, \\
\underline{A} X+\underline{B} Y^{+}-\bar{B} Y^{-}+\gamma X \succ 0,
\end{gathered}
$$

where

$$
\begin{aligned}
\bar{\Psi}_{1}= & {\left[\begin{array}{ccccc}
\bar{\Psi}_{11} & \bar{\Psi}_{12}^{1} & \bar{\Psi}_{13}^{1} & 0 & \overline{\bar{\Psi}}_{15}^{1} \\
* & \overline{\bar{\Psi}}_{22}^{1} & \overline{\bar{\Psi}}_{23}^{1} & 0 & X \bar{A}_{1}^{\top} \\
* & * & \bar{\Psi}_{33}^{1} & \bar{\Psi}_{34}^{1} & 0 \\
* & * & * & \bar{\Psi}_{44}^{1} & 0 \\
* & * & * & * & -\left(2 X-\bar{E}_{1}\right)
\end{array}\right], } \\
\overline{\bar{\Psi}}_{2}= & {\left[\begin{array}{ccccc}
\bar{\Psi}_{11} & \bar{A}_{1} & \overline{\bar{\Psi}}_{13}^{2} & 0 & \overline{\bar{\Psi}}_{15}^{2} \\
* & \overline{\bar{\Psi}}_{22}^{2} & \overline{\bar{\Psi}}_{23}^{2} & \overline{\bar{\Psi}}_{24}^{2} & X \bar{A}_{1}^{\top} \\
* & * & \bar{\Psi}_{33}^{2} & \bar{\Psi}_{34}^{2} & 0 \\
* & * & * & \bar{\Psi}_{44}^{2} & 0 \\
* & * & * & * & -\left(2 X-\bar{E}_{2}\right)
\end{array}\right], } \\
\overline{\bar{\Psi}}_{11}= & 2 \alpha X+\bar{Q}_{1}+\bar{Q}_{3}+\bar{A}^{2}+X \bar{A}^{\top}+\bar{B} Y^{+} \\
& -\underline{B Y}^{-}+Y^{+\top} \bar{B}^{\top}-Y^{-\top} \underline{B} \\
& -\frac{e^{-2 \alpha h_{a}}}{h_{a}}\left(\bar{Z}_{1}+(1-d) \bar{Z}_{3}\right), \\
\bar{\Psi}_{15}^{1}= & \overline{\bar{\Psi}}_{15}^{2}=X \bar{A}^{\top}+Y^{+\top} \bar{B}^{\top}-Y^{-\top} \underline{B},
\end{aligned}
$$

$\overline{\bar{\Psi}}_{i j}=\bar{\Psi}_{i j}, i=2, \ldots, 4, j=1, \ldots, 4$. The controller gains are

$$
\begin{aligned}
& K^{+}=Y^{+} X^{-1}, \\
& K^{-}=Y^{-} X^{-1} .
\end{aligned}
$$

The expressions for $\bar{E}_{1}$ and $\bar{E}_{2}$ are the same as (8) in Theorem 2

Proof. We begin with the $\alpha$-exponential stability of the closed-loop system (22) which is ensured if the LMIs (23), $i=1,2$ hold. We use the fact that $\underline{A} \leq A \leq \bar{A}, \underline{A}_{1} \leq$ $A_{1} \leq \bar{A}_{1}$ and $\underline{B} \leq B \leq \bar{B}$ implies $\bar{\Psi}_{i} \leq \overline{\bar{\Psi}}_{i}$.

Therefore, if $\overline{\bar{\Psi}}_{i}<0$, then $\bar{\Psi}_{i}<0$ for $i=1,2$. Consequently, we have the $\alpha$-exponential stability of the resulting closed-loop system (22) by using the control law $u(t)=\left(K^{+}-K^{-}\right) x(t)$.

To complete the proof, we use the idea of Theorem 3 in order to demonstrate the positivity condition (24). Let the LMI (24) hold. $X^{-1}$ is diagonal positive since so is $X$. Post-multiplication by $X^{-1}$ is applied to the LMI (24). Accordingly, we obtain

$$
\underline{A}+\underline{B} Y^{+} X^{-1}-\bar{B} Y^{-} X^{-1}+\gamma I \succ 0 .
$$

Introducing the expressions for the controller gains $K^{+}$ and $K^{-}$in (25), we obtain

$$
\begin{aligned}
A+B K^{+}- & B K^{-}+\gamma I \\
& \geq \underline{A}+\underline{B} K^{+}-\bar{B} K^{-}+\gamma I \succ 0,
\end{aligned}
$$

for $\gamma \succ 0$.

Moreover, based on Lemma $2, \underline{A}+\underline{B} K^{+}-\bar{B} K^{-}$and $A+B K^{+}-B K^{-}$are Metzler matrices back taking into account that $A_{1}$ and $B$ are simulated to be non-negative matrices. Consequently, the closed-loop system (22) with the parametric uncertainties (3) is also positive.

Remark 3. Compared with Corollary 1, the LMIs proposed in Theorem 2 offer important advantages. First, conditions (23) and (24) take into account the parameter bounds to a greater extent than conditions (17) and (18). In fact, the matrices $\underline{A}, \underline{A}_{1}, \underline{B}, \bar{A}, \bar{A}_{1}$ and $\bar{B}$ are clearly much more employed in Theorem 2 Second, in contrast to the conventional state-feedback control gain which is arbitrary, the controller gain partitioning technique ensures that the two gains $K^{+}$and $K^{-}$are positive. Finally, the results related to the stability and the positivity in the closed-loop derived by this technique are numerically less conservative. This point will be demonstrated in the next section. 


\section{Numerical example}

Consider the delayed system

$$
\left\{\begin{aligned}
& \dot{x}(t)= {\left[\begin{array}{cc}
-1 & 0.5 \\
0.3 & -0.7
\end{array}\right] x(t) } \\
&+\left[\begin{array}{ll}
0.1 & 0 \\
0.3 & 0
\end{array}\right] x(t-h(t)) \\
&+\left[\begin{array}{cc}
0.4 & 0 \\
0.22 & 0
\end{array}\right] u(t), \\
& 0 \preceq h(t) \preceq h_{M} .
\end{aligned}\right.
$$

The parametric uncertainties are defined by

$$
\begin{aligned}
\underline{A} & =\left[\begin{array}{cc}
-1.2 & 0.48 \\
0.25 & -0.75
\end{array}\right], \\
\bar{A} & =\left[\begin{array}{cc}
-0.8 & 0.52 \\
0.35 & -0.65
\end{array}\right], \\
\underline{A}_{1} & =\left[\begin{array}{ll}
0.08 & 0 \\
0.28 & 0
\end{array}\right], \\
\bar{A}_{1} & =\left[\begin{array}{ll}
0.12 & 0 \\
0.32 & 0
\end{array}\right], \\
\underline{B} & =\left[\begin{array}{ll}
0.38 & 0 \\
0.22 & 0
\end{array}\right], \\
\bar{B} & =\left[\begin{array}{ll}
0.42 & 0 \\
0.22 & 0
\end{array}\right] .
\end{aligned}
$$

The objective of this numerical example is to design state-feedback controllers firstly in the form of 21) and then in the form of 15 in order to guarantee the $\alpha$-stabilization of the continuous uncertain system (28) while keeping the states nonnegative. One can remark that the governed system (21) is initially positive. Therefore, Theorem 2 can be easily applied because it can resolve two problems: the first is when the governed system is initially not positive and the second is when it is already positive like in the above example.

Using the Matlab LMI Control Toolbox, when the upper bound of the time-varying delay is $h_{M}=2.04$, it can be easily checked that the LMIs of Theorem 2 are feasible for $\alpha=0.04, d=0.001$ and $\gamma=20$.

The obtained controller gains are

$$
\begin{aligned}
K^{+} & =\left[\begin{array}{cc}
1.9167 & 0 \\
0 & 2.3283
\end{array}\right], \\
K^{-} & =\left[\begin{array}{cc}
2.9372 & 0 \\
0 & 2.3283
\end{array}\right]
\end{aligned}
$$

The closed-loop state matrix is

$$
A+B\left(K^{+}-K^{-}\right)=\left[\begin{array}{cc}
-1.4082 & 0.5000 \\
0.0755 & -0.7000
\end{array}\right]
$$

From the form of the matrix $A+B\left(K^{+}-K^{-}\right)$, one can remark that the controlled system is positive.
The state evolution in opened-loop is shown in Figs. 1 and 2. In turn, the $\alpha$-exponential stability in closed loop for the matrix uncertainties (28) is shown in Figs. 3 and 4. Note that for the two cases of the control gain $K=K^{+}-K^{-}$and $K$ :

- the lower bound of the state $\underline{x}$ is obtained from

$$
\dot{x}(t)=(\underline{A}+\underline{B} K) x(t)+\underline{A}_{1} x(t-h(t)),
$$

- the upper one $\bar{x}$ is obtained from

$$
\dot{x}(t)=(\bar{A}+\bar{B} K) x(t)+\bar{A}_{1} x(t-h(t)) .
$$

Figures 1 and 2 show that the evolution of the system (27) does not satisfy the parametric uncertainties (28). Accordingly, one can remark that $x(t) \geq \bar{x} \geq \underline{x}$.

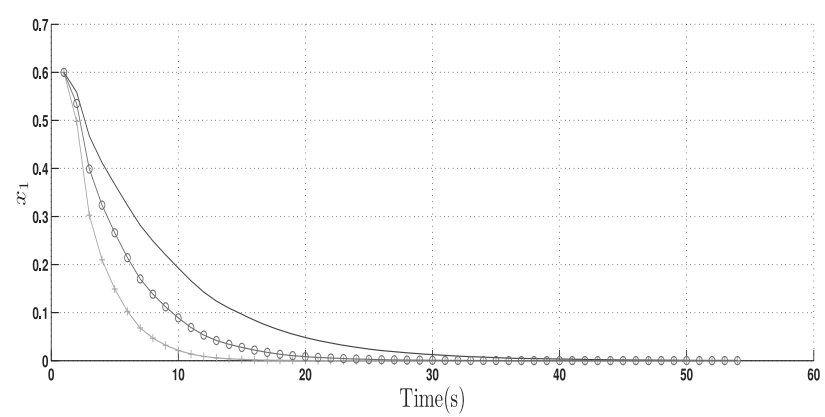

Fig. 1. Evolution of $x_{1}$ in open loop: $z(t)$ (solid line), $\bar{z}$ (open circles), $\underline{x}$ (crosses).

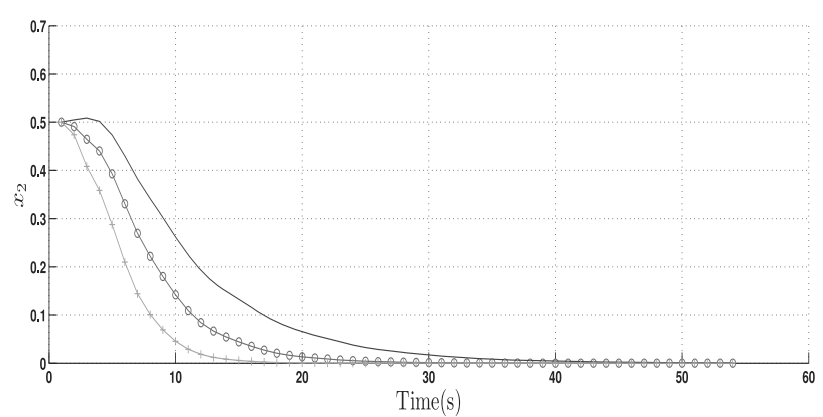

Fig. 2. Evolution of $x_{2}$ in open loop: $z(t)$ (solid line), $\bar{z}$ (open circles), $\underline{x}$ (crosses).

Figures 3 and 4 show that the closed-loop system (27) is robustly $\alpha$-exponential stable for the parametric uncertainties (28) in such way that it evolves boundedly in time between the upper bound of state $\bar{x}$ and the lower one $\underline{x}$. Thus, we obtain $\underline{x} \leq x(t) \leq \bar{x}$. To compare the efficiencies of the control laws proposed in Theorem 2 and in Corollary 1, we have tested various values of $\gamma$ and the 


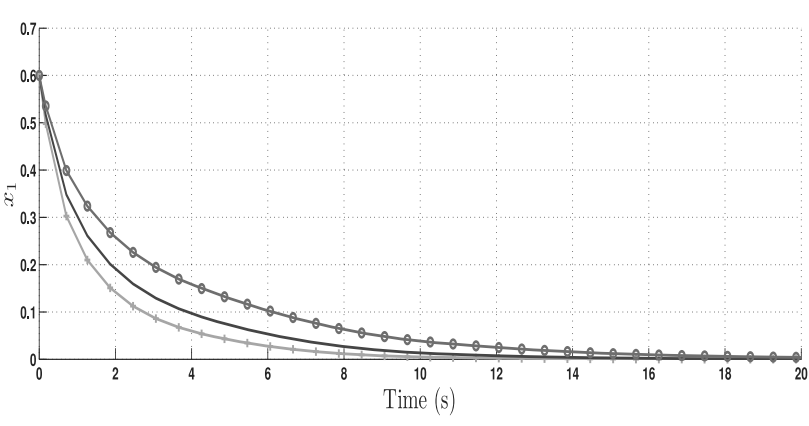

Fig. 3. Robust stabilization of $x_{1}$ for parametric uncertainties 28.

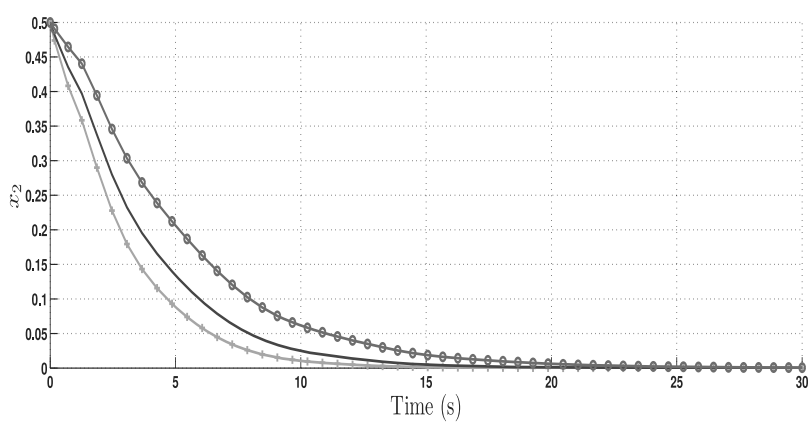

Fig. 4. Robust stabilization of $x_{2}$ for parametric uncertainties 28.

decay rate $\alpha$, the feasibility of the LMIs (23), 24) and the LMIs (17), 18).

Table 1 shows that for different values of $\alpha, d$ and $\gamma$, the maximum delay $h_{M}$ obtained by applying the LMIs of Theorem 2 is greater than the one obtained by applying Corollary 1. Thus, we conclude that the technique of the gain control partition generally allows us to reduce the conservatism.

Using Eqns. (30) and 29, we assume that

- $x(t)$ is represented by a solid line when applying Theorem 2 and by a dashed line when applying Corollary 1

- $\bar{x}(t)$ is represented by the pattern - o when applying Theorem 2 and by - o - when applying Corollary 1.

- $\underline{x}(t)$ is represented by -+ when applying Theorem 2 and by -+- when applying Corollary 1

Figures 5,8 show the evolution of the states of the closed loop system with interval parameter uncertainties (28). It can be seen that the $\alpha$-exponential stability and the positivity in the closed loop are ensured. Moreover, the trajectories are bounded by using Theorem 2 and even Theorem 1 .

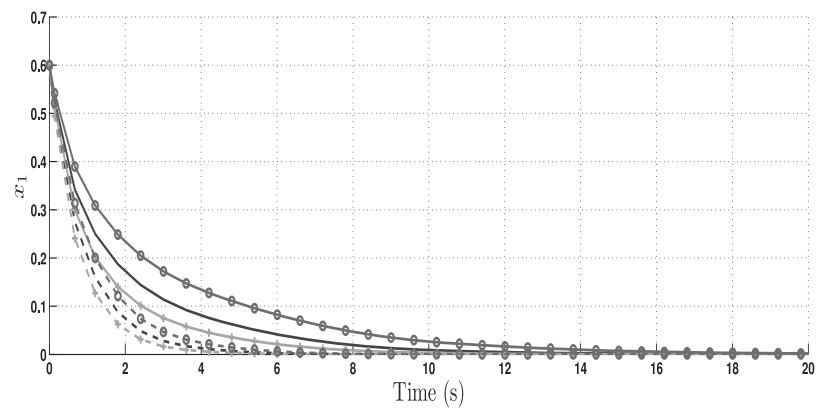

Fig. 5. Robust stabilization of $x_{1}$ for parametric uncertainties 28, $\gamma=20$ and decay rate $\alpha=0.04$.

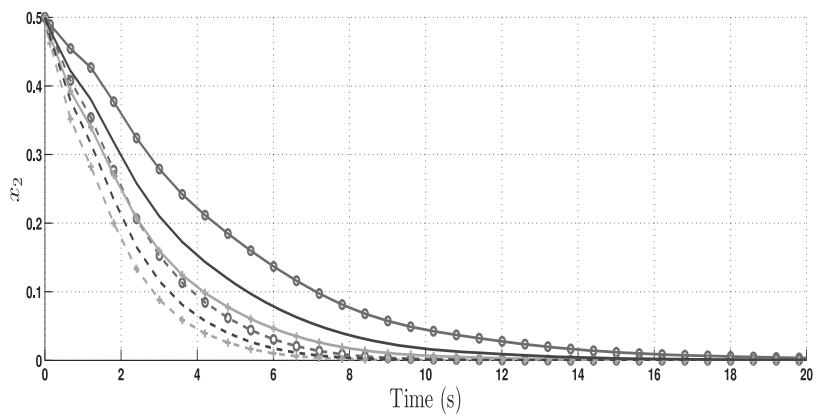

Fig. 6. Robust stabilization of $x_{2}$ for parametric uncertainties 28, $\gamma=20$ and decay rate $\alpha=0.04$.

Remark 4. The delay-dependent conditions proposed in Theorem 2 are more complex than those of Corollary 1 In order to get a simpler form, one can use directly Corollary 1 bearing in mind that it does not reduce conservatism (Table1).

Remark 5. The result proposed in this paper can be extended to the case of a delay $N$-decomposition. In fact, an $\alpha$-exponential stability analysis was made by Elloumi et al. (2015) by using a uniform delay $N$-decomposition technique in order to demonstrate that increasing the number of divisions $N$ allows us to us reduce conservatism. This fact can consequently be used to improve our result.

Table 1. Maximum delay $h_{M}$ of the system 28.

\begin{tabular}{|c|l|l|l|}
\hline & $\gamma=8$ & $\gamma=20$ & $\gamma=25$ \\
& $d=0.1$ & $d=0.001$ & $d=0.001$ \\
& $\alpha=0.1$ & $\alpha=0.004$ & $\alpha=0.007$ \\
\hline \hline Corollary[1 & $h_{M}=3.4$ & $h_{M}=2.7$ & $h_{M}=2.6$ \\
\hline Theorem[2 & $h_{M}=3.8$ & $h_{M}=3.5$ & $h_{M}=3.1$ \\
\hline
\end{tabular}




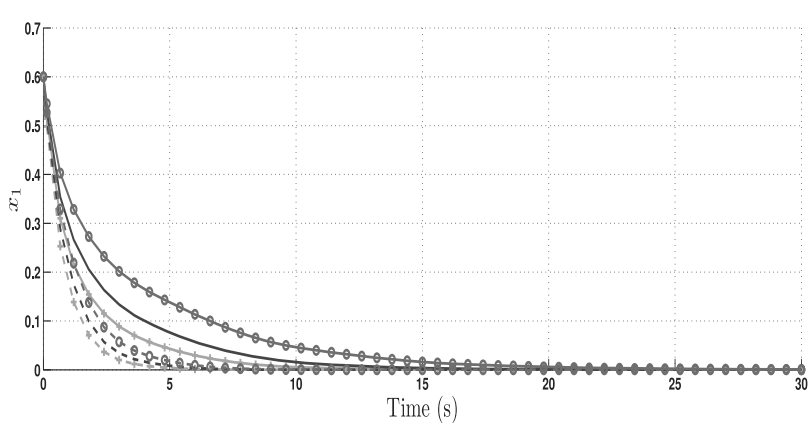

Fig. 7. Robust stabilization of $x_{2}$ for parametric uncertainties (28), $\gamma=8$ and decay rate $\alpha=0.1$.

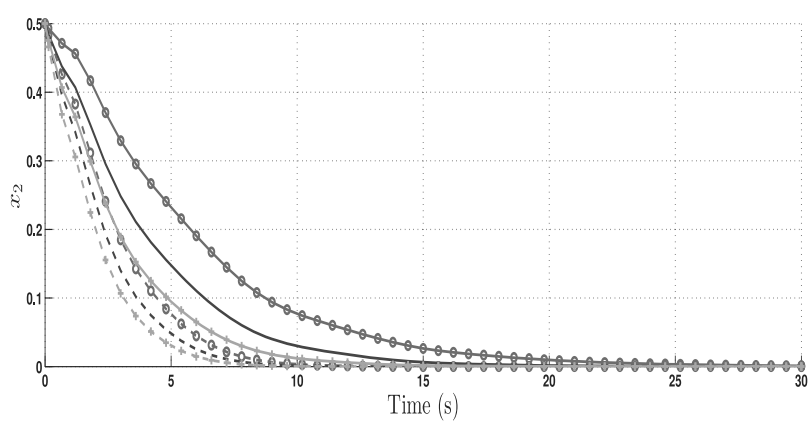

Fig. 8. Robust stabilization of $x_{1}$ for parametric uncertainties (28), $\gamma=8$ and decay rate $\alpha=0.1$.

\section{Conclusions}

This paper provides a uniform bi-decomposition delay approach to the synthesis of robust state-feedback controllers for continuous linear positive time-delay systems. The main idea was based on sufficient conditions for $\alpha$-exponential stability based on a delay $\mathrm{N}$-decomposition technique. Then, when the control design might include parametric uncertainties, this idea was extended to synthesize a state feedback with a partitioned controller gain. Formulated in terms of LMIs, the proposed conditions proposed for the synthesis problems guarantee the exponential stability and the positivity of the state in the closed loop. A numerical example has been treated to illustrate the usefulness of each of the proposed robust state-feedback control laws.

\section{References}

Araki, M. (1975). Application of $m$-matrices to the stability problems of composite dynamical systems, Journal of Mathematical Analysis and Applications 52(2): 309-321.

Bolajraf, M. (2012). Robust Control and Estimation for Positive Systems, Valladolid University, Valladolid.
Chen, X., Chen, M. and Shen, J. (2017). A novel approach to $l_{1}$-induced controller synthesis for positive systems with interval uncertainties, Journal of The Franklin Institute 354(8): 3364-3377.

Elloumi, W., Mehdi, D., Chaabane, M. and Hashim, G. (2015). Exponential stability criteria for positive systems with time-varying delay: A delay decomposition technique, Circuits, Systems and Signal Processing 35(5): 1545-1561.

Farina, L. and Rinaldi, S. (2000). Positive Linear Systems: Theory and Applications, Wiley, New York, NY.

Hale, J. and Lunel, S.M.V. (1993). Introduction to Functional Differential Equations, Springer, New York, NY.

Hmamed, A., Rami, M.A., Benzaouia, A. and Tadeo, F. (2012). Stabilization under constrained states and controls of positive systems with time delays, Mechanical Systems and Signal Processing 18(2): 182-190.

Junfeng, Z., Xianglei, J., Ridong, Z. and Shizhou, F. (2017). Parameter-dependent Lyapunov function based model predictive control for positive systems and its application in urban water management, Control Conference (CCC), Dalian, China.

Kaczorek, T. (2014). Minimum energy control of fractional positive continuous-time linear systems with bounded inputs, International Journal of Applied Mathematics and Computer Science 24(2): 335-340, DOI: 10.2478/amcs-2014-0025.

Kaczorek, T. (2016). Positivity and stability of fractional descriptor time-varying discrete-time linear systems, International Journal of Applied Mathematics and Computer Science 26(1): 5-13, DOI: 10.1515/amcs-2016-0001.

Luenberger, D.G. (1976). Introduction to Dynamic Systems: Theory, Models and Applications, Academic Press, New York, NY.

Mesquine, F., Hmamed, A., Benhayoun, M., Benzaouiaa, A.and Tadeo, F. (2015). Robust stabilization of constrained uncertain continuous-time fractional positive systems, Journal of The Franklin Institute 352(1): 259-270.

Rami, M.A. (2011). Solvability of static output-feedback stabilization for LTI positive systems, Systems \& Control Letters 60(9): 704-708.

Rami, M.A., Tadeo, F. and Benzaouia, A. (2007). Control of constrained positive discrete systems, Proceedings of the American Control Conference, New York, NY, USA, pp. 5851-5856.

Shorten, R., Wirth, F. and Leith, D. (2006). A positive systems model of TCP-like congestion control: Asymptotic results, IEEE Transactions on Networking 14(2): 616-629.

Shuqian, Z., Han, Q.-L. and Zhang, C. (2014). $l_{1}$-gain performance analysis and positive filter design for positive discrete-time Markov jump linear systems: A linear programming approach, Automatica 50(8): 2098-2107.

Zaidi, I. (2015). Robust Stabilization and Observation for Positive Takagi-Sugeno systems, $\mathrm{PhD}$ thesis, Valladolid University, Valladolid. 
Zaidi, I., Chaabane, M., Tadeo, F. and Benzaouia, A. (2014). Static state feedback controller and observer design for interval positive systems with time-delay, IEEE Transactions on Circuits and Systems II 62(5): 506-510.

Zhang, Z. and Yang, H. (2013). Stability and Hopf bifurcation in a three-species food chain system with harvesting and two delays, Journal of Computational and Nonlinear Dynamics 9(2), Paper no.: CND-12-1233.

Zhu, S., Han, Q.-L. and Zhang, C. (2016). Investigating the effects of time-delays on stochastic stability and designing $l_{1}$-gain controllers for positive discrete-time Markov jump linear systems with time-delay, Information Sciences 355(C): 265-281.

Zhu, S., Han, Q.-L. and Zhang, C. (2017). $l_{1}$-Stochastic stability and $l_{1}$-gain performance of positive Markov jump linear systems with time-delays: Necessary and sufficient conditions, IEEE Transactions on Automatic Control 62(7): 3634-3639.

Zhu, S., Meng, M. and Zhang, C. (2013). Exponential stability for positive systems with bounded time-varying delays and static output feedback stabilization, Journal of The Franklin Institute 350(3): 617-636.
Wafa Elloumi received her $\mathrm{PhD}$ degree in electrical engineering in 2017 and her MSc degree in automatic control and industrial computing in 2012 from the University of Sfax, Tunisia. Currently, she is an associate researcher in the Laboratory of Sciences and Techniques of Automatic and Computer Engineering (Lab-STA) at the National Engineering School of Sfax (ENIS), University of Sfax. Her research interests include stability and stabilization, as well as time-delay and positive systems.

Mohamed Chaabane was born in 1961. He received the $\mathrm{PhD}$ degree in electrical engineering from the University of Nancy, France, in 1991. He is currently a professor with the National School of Engineering, University of Sfax, where he has been a researcher with the Laboratory of Sciences and Techniques of Automatic Control and Computer Engineering (Lab-STA) since 1997. From 1988 to 1992, he was an associate professor with the University of Nancy, working as a researcher at the Center of Automatic Control of Nancy (CRAN). His main research interests are in the field of robust and optimal control, fault tolerant control, delay systems, descriptor systems, fuzzy logic systems and applications of these techniques to fed-batch processes, asynchronous machines, agriculture systems and renewable energy.

Driss Mehdi received an engineer degree from Mohammadia Engineering School, Rabat, Morocco, in 1979 and a PhD degree in automatic control from Nancy University in 1986. From 1988 to 1992 he was a senior lecturer at Louis Pasteur University in Strasbourg, and since 1992 he has been a professor at the University of Poitiers. His main interests are in robust control, as well as delay and descriptor systems.

Received: 6 May 2017

Revised: 10 October 2017

Re-revised: 18 January 2018

Accepted: 7 April 2018 